\title{
A Review of RPL Objective Function based Enhancement Approaches
}

Ines Kechiche* Ines Bousnina, Abdelaziz Samet

SERCOM Laboratory, Tunisian Polytechnic School, University of Carthage, La Marsa 2078, Tunisia

A R T I C L E I N F O

Article history:

Received: 24 July, 2020

Accepted: 02 September, 2020

Online: 14 September, 2020

\section{Keywords:}

$R P L$

LLNS

Routing

Objective function

Metric combination

Fuzzy logic

\begin{abstract}
A B S T R A C T
Since the release of of the IPv6 Routing protocol for Low-Power and Lossy Networks by the IETF ROLL working group, several enhancement schemes were proposed. In fact, They aim to extend the network lifetime, reduce congestion, mitigate end to end delay and moderate energy consumption. In fact, considering the vast area of Low-Power and Lossy Networks applications, the routing protocol was designed with a great deal of flexibility without dictating any specific routing metric/constraint to be used for building the routing topology. This paper presents a deep review of recent works on Routing Protocol for Low-Power and Lossy Networks and highlights Objective Function enhancements scheme. The objective is to provide an insight into relevant efforts such as novel metrics design and fuzzy logic techniques used for the Objective Function metric combination. The proposed enhancement schemes, highlight some limitations and give useful guidelines for future developments are also discussed.
\end{abstract}

\section{Introduction}

The IPv6 routing protocol for low-power and lossy networks (LLNs), was designed to achieve routing function for resource-constrained devices in industrial, home, and urban environments [1]. Since routing is of great apprehension in spoiling the resources in these devices, poor path selection may cause the scant assets to drain out quickly. Thus, RPL could achieve what traditional routing protocols such as OSPF, OLSR and AODV or DSR failed to do and overcome LLNS networking limitations, such as high loss rates, low data rates, and network instability [2].

RPL depends on objective function (OF) operations to choose a preferred parent for traffic forwarding. The OF considers a number of nodes/networks metrics and constraints to select the best path. Two OFs were dressed by the IETF ROLL working group, namely OF zero (OF0) [3] and Minimum Rank with Hysteresis Objective Function (MRHOF) [4]. The primitiveness of the standardized OFs have motivated OF refinement work to seek for enhancing network performance. Hence, many research works have tackled this issue and focused on OF and new metric calculation strategies that seek to improve network performances in terms of energy, reliability, latency and lifetime. Although the main line of OF optimization was multiple metrics combination many enhancement schemes resort to Cross-layer design to tap mac layer information and bring out the fitting measurements that would build the optimal OF.

*Corresponding Author: Ines Kechiche, Email: ines.kechiche@gmail.com

\subsection{Contribution of the Survey}

Through this survey, the goal is to provide the research community with a solid piece of work that gathers all research efforts made for RPL OF enhancement. Our survey will help them get a deep vision on existing contributions and their shortcomings. Through enhancement strategies classifications, introduced nodes and networks metrics not specified in [5] are highlighted. Thus, researchers working on this issue will find at their disposal all the necessary information on already introduced metrics as well as combination techniques applied such as simple weighted or Artificial Intelligence (AI) based association in addition to the routing metrics taking part of these operations. As a matter of fact, in this survey, we do not settle for listing OF improvement work only but also classify them and outline plausible areas for follow-up research.

\subsection{Organization of the Survey}

This paper surveys substantial contributions brought by the research community to develop RPL routing in LLNs. An overview of various efforts done on optimizing the RPL OFs is presented. Our goal is to label the different approaches used to tackle this issue by stressing on introduced routing metric and presenting different metric combination techniques. This paper also brings out the fitting metrics that could result in RPL enhancement. This survey is organized as follows. First, section 2 recalls RPL basic knowledge and highlights relevant aspects of this protocol namely Destination- 
Oriented Directed Acyclic Graph (DODAG) construction process, communication pattern and OF operation. Then, section 3 focuses on main RPL OF based enhancement strategies. In section 4, the strengths and weaknesses of the aforementioned enhancement proposals, as well as relevant open issues and future work guidelines are discussed. Section 5 presents enhancement opportunities for future works. Finally, section 6 concludes the paper.

\section{The IPv6 Routing Protocol for LLNS}

RPL [1] is a distance-vector and a source routing protocol designed by the ROLL Working Group through RFC 6550 in order to meet specific requirements of LLNs. It is an IPv6 ready routing protocol based on a 6lowpan adaptation layer that allows micro devices to take part of the Internet of Things (IoT). Two basic components of RPL are the DODAG and the OF. Thus the RPL protocol purpose is to attenuate the cost of reaching the root node from any node in the network. This cost calculation is handled by RPL OF.

As it points collection-based networks, it supports three traffic patterns: multipoint-to-point traffic (MP2P), point-to-multipoint traffic (P2MP), and point-to-point $(\mathrm{P} 2 \mathrm{P})$ traffic.

\subsection{DODAG Building Process}

Rooted at an LLN border router (LBR), the DODAG provides RPL with an accurate vision of the network status and then it is easy to determine low cost paths to address any node from root. It is built on the basis of the parent selection process through what each node selects a preferred parent (best path) to transfer its packets toward the root node or other nodes. Thereby, both upward and downward traffic are enabled.

\subsubsection{ICMPv6 messaging}

When activated, RPL discards the IPv6 Neighbor Discovery process [6]. DODAG is then maintained through RPL ICMPv6 messages (i.) DAG Information Solicitation (DIS) used by non root nodes to persue an active RPL instance, (ii.) DAG Information Object (DIO) initially flooded by DAG root to advertise the creation of an RPL DAG and then relayed by nodes joining the DAG (iii.) Destination Advertisement Object (DAO) messages are used for parent selection, confirming or canceling processes. They allow reverse route information by keeping track of visited nodes along the upward path from leaf node to DODAG root. Thus, each node, except the DODAG root, sends a DAO message to populate the routing tables with prefixes of their children and to announce their addresses and prefixes to their parents. A Destination Advertisement Object Acknowledgment (DAO-ACK) is then sent as a response by the DAO recipient, i.e. the preferred parent.

\subsubsection{Communication pattern}

Basically RPL supports different Modes of OPeration (MOP) [1] namely (i) non-storing MOP without or with downward traffic, respectively MOP0 and MOP1, (ii) storing MOP without or with multicast support, respectively MOP2 and MOP3. Fig. 1 illustrates traffic forwarding in case of storing and non-storing modes. Storing mode makes nodes produce less networking overhead. However, the non storing mode is interesting since nodes with strict memory limitations could not store a large number of routing states. When non-storing mode (MOP 0) is enabled, intermediate routers (ie: non-BR nodes) do not keep track of available routes and downward routes are not maintained. However the LBR may resort to source routing whenever it needs to direct the traffic along a specific path. In (MOP 1), downward routes are enabled, hence P2P and MP2P communication is allowed. However, route calculation is performed at the DODAG root and subsequently forwarded to its destination. In (MOP 2), downward routes are also supported, but differently from MOP 1. Through DAO messages exchange, the intermediate nodes maintain, individually, a routing table and no longer resort to the root node to make downward traffic possible. Finally MOP 3 extends MOP 2 operation by the possibility of multicasting data. So far non-root are able to address enabled clusters of nodes through multicast DAOs. According to whether nodes are multicast ready or not, the MOP flag is set to 3 or 2 subsequently. For better comprehension of communication pattern related issues [7] is advised.

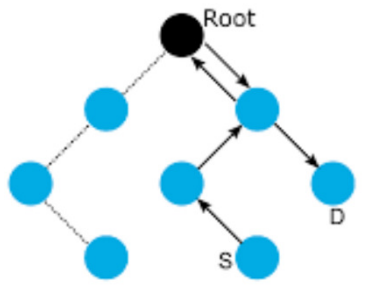

(a) RPL non-storing mode

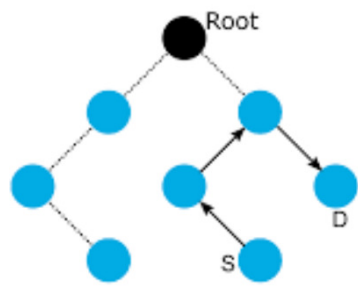

(b) RPL storing mode
Figure 1: $\mathrm{P} 2 \mathrm{P}$ communication pattern for MOP 1 and MOP 2 [8]

\subsubsection{Trickle algorithm}

Since DIO messages exchange may lead to raising traffic overhead, an adaptive beaconing scheme is used for RPL routing signaling: the trickle algorithm [9, 10]. In trickle-based strategy, transmission of DIO messages is dynamically adjusted. When inconsistencies are detected (i.e. a node's data is incoherent with its neighborhood), the communication rate exponentially increases to resolve quickly the inconsistency. Otherwise, nodes slow down communication until reaching a predefined maximum value. However, this may affect RPL ability to swiftly respond to topology changes [6].

\subsection{Objective Function Operations}

RPL OF handles next hop selection issues. In fact, as depicted in Fig. 2, the OF defines, (i.) how to estimate the path cost, (ii.) how to pick out parents (when, who, how many), (iii.) how to estimate the rank and (iv.) how to propagate the path cost. To address the cited issues, the OF defines how a node transcribes one or more metrics into a rank value that represents the routing distance from a node to a LBR. Thus, the selected metrics evaluate path cost and hence decide about the best path. Some RPL implementations rely on a single metric while other ones merge various routing metrics and 
constraints [10]. Depending on their features, the routing metrics are organized on link metrics and node metrics. They may reflect quality or quantity, and be static or dynamic. In addition to routing metrics OF parents may consider some routing constraints while taking routing decisions such as avoiding unreliable links or nodes with low power level battery. The arrangement of the aforementioned metrics and constraint may pertain to implementation needs.

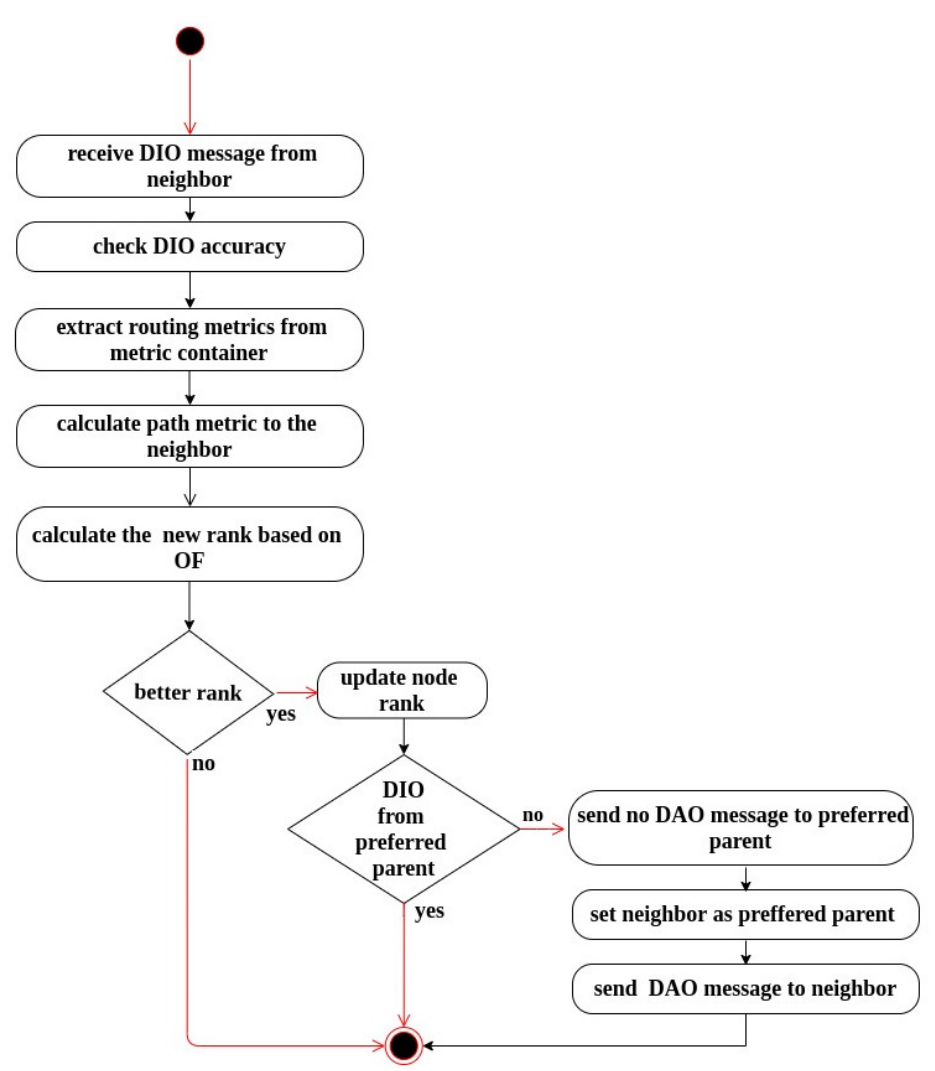

Figure 2: RPL OF main operations

\section{The IPv6 Protocol for LLNs Objective Function Enhancement Strategies}

Compelled by the challenging needs of LLNs, a collection of routing metrics with static or dynamic constraints [2], the IETF-ROLL working group designed RPL. However, the choice of the two settings; used metrics and constraints combinations and layer were left as an open issue for ulterior refinement work since RPL may have vast areas of applications. So far, depending on applications scenarios [11] and target environments requirements, many adaptation and improvement work have been made. Some of them are based on improving the metrics used for constructing the routes, others focused on MAC protocols overhaul. RPL built routes according to a parent selection process performed by an Objective Functions (OF). To supply different LLNs application requirements, a collection of routing metrics with static and dynamic constraint, have been introduced in [5]. Choice was left to the OF implementer to decide how to express each metric, to develop composite metrics or to define new ones. In the following, recent works in this direction are investigated. A summary of the reviewed OFs goals and specification is provided through tables 4 and 5 .

\subsection{Single Metric Based OF}

Till now, only two basics OFs are published by the IETF Roll working group namely, OF0 [3], and MRHOF [4]. Rank calculation is based on Hop Count (HC) metric for OF0 and Expected Transmission Count (ETX) metric in the case of MRHOF. Nevertheless ETX and HC metrics seem clearly insufficient to deal with common networking issues such as throughput, energy saving and load balancing,

To deal with issues related to varying traffic patterns, ETX metric calculation method was revised in [12]. Bearing in mind that link quality estimation should not rely only on upward traffic, authors enable the parent node link quality information piggybacking through downward traffic. The proposed DT-RPL nodes will henceforth stabilize the ETX metric faster than with standard RPL nodes. Authors compared their scheme to standard RPL using different traffic patterns and demonstrate that they perform better in both Packet Delivery Ratio (PDR) and control overhead. In [13], authors proposed a mac -aware routing metrics namely R-metric and Q-metric. The R-metric can be used for reliability purpose and it measures end-to-end reliability regarding MAC layer measured back-offs and retransmissions. Thus, with R-metric the estimation of this probability is faster than the ETX estimation. The Q-metric is advised when it is a matter of improving the network lifetime by selecting the lightest parent, in terms of traffic load. The authors also boosted their proposed OF by adapting the corresponding MAC parameters so as to mitigate nodes energy consumption in the network. Unfortunately, for their proposed scheme evaluation, the author considered the back pressure protocol, a non RPL-protocol.

Authors in [14] substituted the ETX metric by another metric that decreases measured delay on path leading to root node. Their proposed Averaged Delay (AVG_DEL) metric gather all hop by hop measured delays along the path from a node towards the DAG root. Authors did some changes on MAC-layer to make learning neighbors wake-up phases possible and thus compute the AVG_DEL. Although promising performances against ETX based OF the proposed solution Simulation results did not present its impact on network scalability and energy consumption. Another delay based RPL OF enhancement approach was presented in [15]. First the authors developed a novel real-time and end-to-end delay EED estimation mechanism considering both path delays and node processing delays. According to the authors, the proposed EED is more accurate than the Expected Transmission Time ETT based. Afterwords, authors presented an RPL Adaptation for EEDEM (RAEEDEM). They claim their proposed scheme enhanced slightly the Packet Reception Ratio PRR (14\%) and measured Average EED by $(12 \%)$.

A new routing metric, TXPFI, was introduced in [16]. It metric estimates the expected number of frame retransmission needed till data correct transmission. Author claims that applied to the 
IETF RPL protocol, the novel metric achieves a low packet drop rate and proves its efficiency to get around non cooperative relays. Instead,authors in [17] tried to maximize the network lifetime by selecting next hop to DODAG root according to node remaining energy. Beginning from node energy, nodes add up time periods spent in each state or activity (transmit, receive, idle,sense, compute). Thus, the remaining energy capacity can be easily computed according to a formula specified in devices datasheet. The measured path cost will then correspond to the remaining energy of the weakest node on the path. According to the proposed OF, nodes will then select a neighbor for which path to root node held the best minimum lifetime value. The major inconvenience of this method is an observed degradation of the transmission reliability since inefficient routes with bad radio links may be used. In another work [18], authors proposed a new approach where they worked on increasing network reliability while maintaining a balanced energy consumption among network nodes. First, they tried to spot energybottleneck nodes and then balance traffic load between them. To this end, authors designed the Expected LifeTime (ELT) metric to estimate how much time a node has to live until its energy starvation. According to simulation results the proposed OF ensured a balanced energy consumption for topology nodes independently from their distance the DODAG Root. However, an additional latency is observed.

Different from previous works, authors in [19] and [20] tackled the problem of unbalanced traffic load by considering the number of children. In [19] a load balanced OF (LB-OF) that aims to balance workload distribution among all nodes in LLNs specially bottleneck nodes is proposed. To this end, authors substituted ETX metric by considering the children number for rank calculation and hence preferred parent selection. Compared to MRHOF, the proposed strategy helped to balance the number of children nodes for the overburdened nodes and thus ensure node lifetime maximization. However, authors did not study the proposed OF impact on PDR or end-to-end delay. Authors in [20] called their proposed solution Bounded Degree RPL(BD-RPL). Rather than considering the children number as a metric they used it as a constraint for DODAG construction by setting boundaries for the number of children a preferred parent could accept. Thus, the resulting DODAG is called a k-degree tree. Owing to authors the profit of the proposed scheme is the absence of any additional overhead compared to RPL. It is also independent from the radio link quality metric. According to authors, BD-RPL (with degree 3) achieved an improvement over standard RPL by an average of $10 \%$ in packet delivery, $50 \%$ in energy consumption, and $60 \%$ in delay. For load regulation purpose, authors in [21] introduced a new node metric for RPL based on the estimated node Queue Backlog in order to ensure better throughput performance while maintaining usual delay values and be suitable for different network appliances. Table 1. Provides a summary of the aforementioned enhancement schemes.

\subsection{Metric Combination Based OF}

Considering a single routing metric for the OF operations seems to be insufficient to fulfill LLN application requirements. For example, focusing only on network reliability enhancement may be harmful for the network expected life time and latency. Thus, to overcome these limitations, many researchers worked on associating new or existent routing metrics and constraints in order to achieve better networking performances. While some of the enhancement scheme focused on simple weighted combination of RPL usual metrics [21][25] specified by [5], others [26]-[31] resorted the Fuzzy Logic (FL) properties to address the choice of the best path making possible the combination of non additive metrics.

\subsubsection{Additive metric based routing strategies}

To deal with issues related to traffic load and congestion, the author in [21] presented also an enhanced version of their proposed queue backlog based OF where they resort to associate the Queue Backlog node metric to other metrics from those proposed in [5]. Thus, the node weight is a scalar association of a penalty function trading system queue occupancy that authors try to minimize and a link usage cost computed through ETX metric ( as presented in [21]) or any other metric from those cited in [5] if needed. In the same way, authors in [22], presented a new RPL OF called Congestion-Aware OF (CA-OF). This OF implies in addition to ETX on a new RPL routing metric not introduced in [5] namely the Buffer Occupancy (BO). The aforementioned OF aims to enhance traffic reliability by selecting parents across less congested paths. The resulting additive metric applies adaptive weights to ETX and BO metrics depending on traffic intensity. Thus, in low traffic the proposed OF stands on the ETX metric to select a preferred parent. As soon as the network tends to be congested, the ETX metric is dismissed and the OF considers only the $\mathrm{BO}$ for the next hop election process. According to authors, CA-OF could achieve good network performance regarding reliability, energy consumption and throughput. In [23], authors worked on improving the end-to-end packet delivery performance by regulating the traffic load within the routing tree. Thus, they proposed QU-RPL, to deal with traffic congestion problems for parent selection, the authors introduced a novel metrics. The queue utilization (QU) coefficient measured for a node $\mathrm{k}$ ensures the network stability by avoiding inefficient parents changes authors worked on setting a threshold to recognize when the network is congested and consequently associate the QU factor to HC and ETX metric in order to designate a preferred parent.

Singh and Chen introduced a new OF for efficient routing (OFER) [24] based on a composite efficient routing (CER) metric used for best parent selection. The CER metric combines a set of weighted metrics, namely, the link quality ETX, packet loss due to queue occupation, node's remaining time to live, delay and observed bottlenecked nodes. Authors claim that OF-ER could not only reduce energy consumption and queue loss, but also expand node's expected lifetime. Unfortunately, the authors did not report the introduced metric impact on traffic overload nor its ability to support network scaling

Authors in [25] Improved RPL (I-RPL) where the OF relies on an LCI index for parent node selection. The LCI index is deducted from various metrics, for instance, link quality and node energy. Hence, for preferred parent selection, the next-hop with the largest LCI index is selected. However, I-RPL stores also a potential parent node that could be used to forward traffic if multi-path routing is needed to relieve congested nodes. According to the authors, the proposed scheme achieved promising results in terms of load 
Table 1: Single metric based Objective Functions

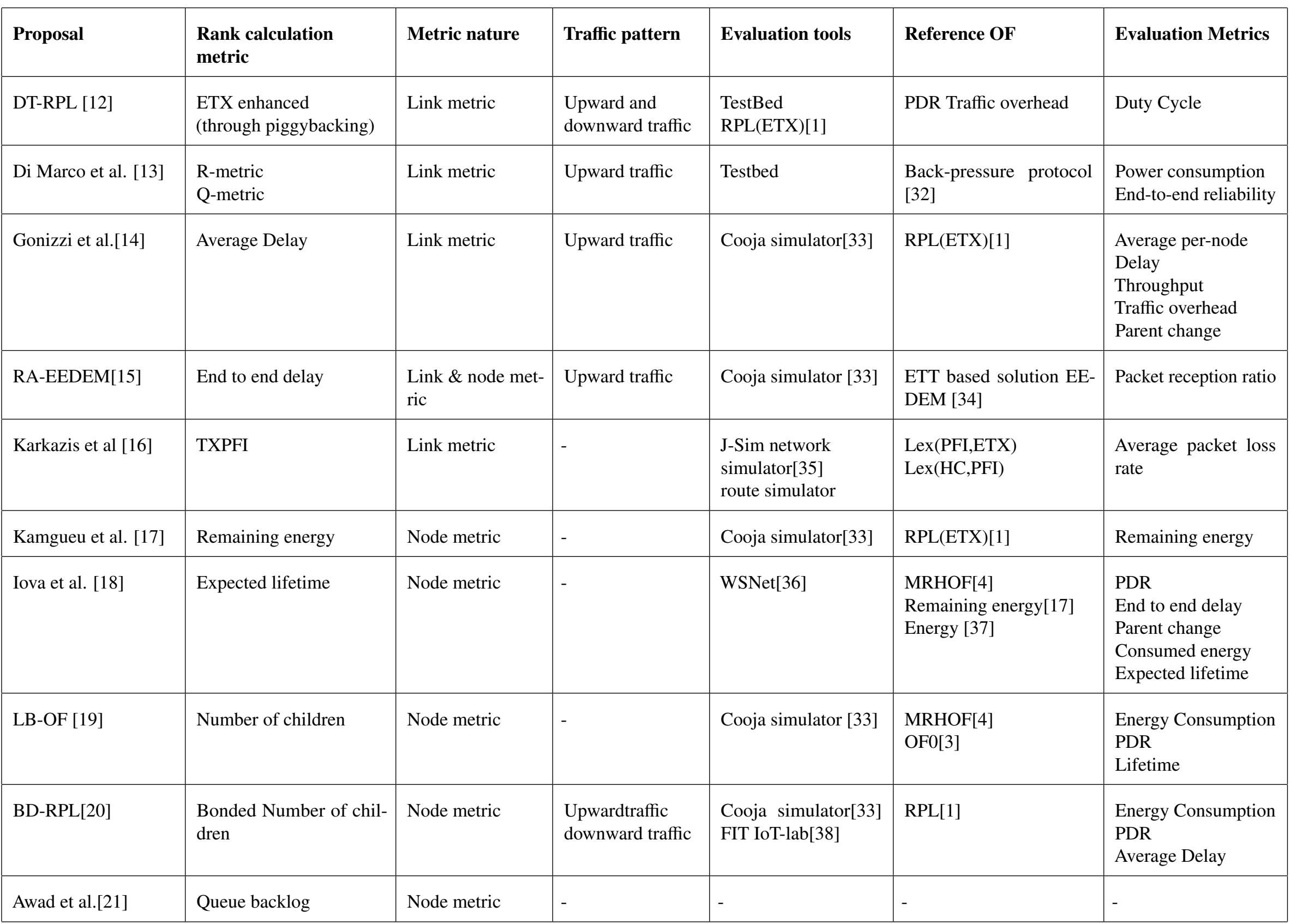


Table 2: Weighted Metric Combination based OF enhancement schemes

\begin{tabular}{|l|l|l|l|l|l|l|}
\hline Proposal & $\begin{array}{l}\text { Link metrics } \\
\text { used }\end{array}$ & node metrics used & $\begin{array}{l}\text { metric } \\
\text { combination }\end{array}$ & $\begin{array}{l}\text { Evaluation } \\
\text { tool }\end{array}$ & Reference OF & Evaluation metric \\
\hline Awad et al.[21] & ETX & Queue backlog & Scalar addition & - & - & - \\
\hline CA-OF [22] & ETX & Buffer Occupancy & $\begin{array}{l}\text { Weighted Combi- } \\
\text { nation }\end{array}$ & $\begin{array}{l}\text { Cooja sim- } \\
\text { ulator [33] }\end{array}$ & $\begin{array}{l}\text { RPL(ETX) } \\
\text { RPL(Energy) } \\
\text { OF0 }\end{array}$ & $\begin{array}{l}\text { Buffer Overflow } \\
\text { Loss packet } \\
\text { Throughput } \\
\text { Energy consumption } \\
\text { Packet delivery ratio }\end{array}$ \\
\hline Qu-RPL [23] & ETX & $\begin{array}{l}\text { Hop Count Queue } \\
\text { utilization }\end{array}$ & $\begin{array}{l}\text { Weighted Combi- } \\
\text { nation }\end{array}$ & $\begin{array}{l}\text { Real } \\
\text { testbed }\end{array}$ & Tiny RPL & $\begin{array}{l}\text { Packet delivery } \\
\text { Routing overhead } \\
\text { Parent change }\end{array}$ \\
\hline En-RPL [24] & ETX Delay & $\begin{array}{l}\text { Packet loss Life- } \\
\text { time queue uti- } \\
\text { lization Number } \\
\text { of bottlenecked } \\
\text { nodes }\end{array}$ & $\begin{array}{l}\text { Weighted Combi- } \\
\text { nation }\end{array}$ & $\begin{array}{l}\text { Cooja sim- } \\
\text { ulator [33] }\end{array}$ & $\begin{array}{l}\text { Standard RPL } \\
\text { with qu-RPL } \\
\text { congestion } \\
\text { avoidance } \\
\text { configuration }\end{array}$ & $\begin{array}{l}\text { Queue loss ratio } \\
\text { End to end delay } \\
\text { Traffic overhead } \\
\text { Energy consumption }\end{array}$ \\
\hline IRPL[25] & ETX & $\begin{array}{l}\text { Data Throughyut } \\
\text { Data TX Rate }\end{array}$ & $\begin{array}{l}\text { Weighted Combi- } \\
\text { nation }\end{array}$ & $\begin{array}{l}\text { Cooja sim- } \\
\text { ulator [33] }\end{array}$ & $\begin{array}{l}\text { RPL(ETX) } \\
\text { RPL(Residual } \\
\text { Energy) }\end{array}$ & $\begin{array}{l}\text { Data delivery rate } \\
\text { Residual Energy } \\
\text { Parent changes } \\
\text { End to end delay }\end{array}$ \\
\hline
\end{tabular}

balance, end-to-end delay and packet delivery.

The aforementioned enhancement schemes are summarized in Table 2.

\subsubsection{Fuzzy logic based routing strategies}

Fuzzy logic [26] is a heuristic method based on artificial intelligence process that was widely implemented to deal with complexity problems in communication and computer networks. In [27], authors employed fuzzy logic to gather heterogeneous routing metrics namely delay, ETX and energy into one neighbour quality value. Through a two stage fuzzification engine, they estimated first the quality of service (QoS) from delay and ETX. Then, they combined obtained QoS to energy in a second fuzzification stage to estimate neighbors' resulting quality. Authors claim that their proposed fuzzy logic based OF reduced packet loss and parents change than ETX based RPL. In [28], a designed QoS-aware fuzzy logic OF (OFFL) is presented. Authors stressed out the profit of various metrics association to meet routing requirements and selected $\mathrm{HC}$, end to end delay, ETX and battery level for their fuzzification engine. The proposed solution, performed better energy conservation and end to end delay than OF (ETX) especially for distant nodes. In [29], an OF based on a combined metric using fuzzy logic method OF-EC is proposed. Similar to [27], they selected ETX, HC and energy metrics as input for the fuzzy logic process but computed energy consumption differently. Thus, to determine the energy consumption they used both transmission and reception consumed power, processing power and both full and low power modes consumption. Authors claim that the proposed OF-EC showed satisfying performance for measured PDR, network lifetime, convergence time, latency, overhead, and energy consumption compared to other RPL OF namely OF-FUZZY [27], MRHOF and ENTOT [39].

Authors proposed in [30] the Composite Metric OF (CMOF) that combines weighted latency and ETX path metrics expecting to enhance both delay and PDR by selecting paths with good connectivity and less traffic. In fact, while ETX will grant the use of reliable links, latency will measure both traffic and contention at any given node by summing time packets spent in the transmit queue and the time taken to access the channel. Moreover, authors employed a power regulation scheme. CMOF stands apart from similar works by substituting default values used for fuzzy sets by new thresholds unlike [28]. Nevertheless, the authors did not defend the choice of these new values nor explain the accuracy of the used thresholds.

An extra scheme of metrics combination using fuzzy logic method is given in [31], the authors presented a holistic OF, OPP-FL, that combines several representative metrics to enhance best route selection namely ETX, HC and Children Number (CN) making use of the fuzzy logic approach.Through the proposed OF they tried to limit the impact of a node overload on routing performance and equilibrate the routing load between the different parent nodes while trying to stay closest to the root. Indeed, the number of children nodes attached to a parent can negatively affect the delay of data transmission in the network. The work focused on delay and reliability enhancement by promoting the selection of parents who are closer to the root and less burdened. Simulation results showed that OPP-FL outperformed the standard ETX based OF (MRHOF) and the fuzzy logic based OF OF-EC in terms of PDR and network latency in high network density topologies. Authors proposed in [40] a fuzzy-based mobility OF (FMOF) through which they apply fuzzy logic process to a hand-off enabled RPL mechanism, the mRPL [41], in order to reinforce mobility support in WSN networks. As input for the fuzzification engine, the authors selected three metrics namely HC, ETX, and Radio Signal Strength Indicator (RSSI). However, different from previous enhancement schemes, the authors tested different input weighted combinations. According to simulation results, the proposed scheme achieved good results in terms of reducing the handoff delay and PDR in case of low 
Table 3: Fuzzy logic based OF enhancement schemes

\begin{tabular}{|c|c|c|c|c|c|}
\hline Proposal & Link metrics used & Node mectrics used & Evaluation tool & Reference OF & Evaluation metric \\
\hline Kamgueu et al.[27] & ETX Delay & Energy & $\begin{array}{ll}\text { Real } & \text { indoor } \\
\text { testbed } & \end{array}$ & RPL(ETX) & $\begin{array}{l}\text { Power consumption } \\
\text { End to end delay } \\
\text { Parent changes } \\
\text { loss rate }\end{array}$ \\
\hline OF-FL[28] & $\begin{array}{l}\text { end to end delay } \\
\text { ETX }\end{array}$ & $\begin{array}{l}\text { Hop Count } \\
\text { Battery level }\end{array}$ & $\begin{array}{l}\text { Cooja simulator } \\
{[33]}\end{array}$ & $\begin{array}{l}\text { MRHOF [4] } \\
\text { OF0 [3] }\end{array}$ & $\begin{array}{l}\text { Power Consumption } \\
\text { End to end delay } \\
\text { Parent changes } \\
\text { Average Hop count } \\
\text { Loss rate }\end{array}$ \\
\hline OF-EC [29] & ETX & $\begin{array}{l}\text { Hop Count } \\
\text { Energy consumption }\end{array}$ & $\begin{array}{l}\text { Cooja simulator } \\
{[33]}\end{array}$ & $\begin{array}{l}\text { RPL(ETX) } \\
\text { EN-TOT } \\
\text { OF-Fuzzy } \\
{[27]}\end{array}$ & $\begin{array}{l}\text { Power consumption } \\
\text { Traffic overhead } \\
\text { Packet delivery ration }\end{array}$ \\
\hline CMOF [30] & ETX & Packets latency & $\begin{array}{ll}\text { Cooja simulator } \\
\text { [33] }\end{array}$ & MRHOF [4] & $\begin{array}{l}\text { Per hop delay } \\
\text { Packet delivery ratio }\end{array}$ \\
\hline FMOF [40] & $\begin{array}{l}\text { ETX } \\
\text { RSSI }\end{array}$ & Hop count & $\begin{array}{l}\text { Cooja simulator } \\
\text { [33] }\end{array}$ & $\begin{array}{l}\text { MRHOF [4] } \\
\text { OF0 [3] }\end{array}$ & $\begin{array}{l}\text { Hand-off delay } \\
\text { Packet reception ratio } \\
\text { Traffic overhead }\end{array}$ \\
\hline OPP-FL [31] & ETX & $\begin{array}{l}\text { Hop Count } \\
\text { Children number }\end{array}$ & $\begin{array}{l}\text { Cooja simulator } \\
\text { [33] }\end{array}$ & $\begin{array}{l}\text { MRHOF [4] } \\
\text { OF-EC [29] }\end{array}$ & $\begin{array}{l}\text { Average end to end } \\
\text { delay } \\
\text { Traffic overhead } \\
\text { Packet delivery ratio }\end{array}$ \\
\hline
\end{tabular}

transmission rate. Table 3. Summarizes the surveyed fuzzy logic based OF enhancement schemes.

\section{Discussion}

The previous section reviewed relevant and recent approaches for OF based RPL enhancement strategies. According to the results presented in each work, the majority of aforementioned solutions could improve the PDR, reduce congestion and preserve energy consumption. Although these approaches aimed to improve the standard RPL, we deem that the ability of the proposed solution to supply to IoT application requirements is still to be proven, since the performances of the mostly proposed schemes were compared to standard RPL and there are still a need for further testbed validation. The following are some findings with regards to the reviewed enhancement schemes.

\subsection{Metric Combinations Approach}

Relying on a single metric for OF rank calculation seems to be deficient and in most cases ends up degrading others while ensuring interesting performances in terms of some network parameters. Our main purpose of reviewing single metric based OF was to point out novel metrics not yet cited in [5]. Nevertheless, metric combination could achieve promising results especially if we resort to cross-layer design. AI based techniques should be more exploited not only through fuzzy logic combination processes but also for weighted metric combination. Since it would be so helpful for researchers to decide for the most suitable metric weights values.

\subsection{Energy Efficiency}

Energy efficiency is among key considerations of the reviewed OF since it was oftenly present either as OF metric or as a node constraint. In fact, most of the reviewed enhancement schemes aimed at preserving energy while providing a good PDR. Simulations have shown that used as a single metric, the node energy is not that efficient and should be associated with other metrics to provide promising results.

\subsection{Multipath Routing and Congestion Avoidance}

Congestion avoidance was among main $\mathrm{OF}$ enhancement goals since it would affect both end to end delay and network reliability. Several research works resort to multipath routing to consolidate congestion avoidance efforts and decrease the number of bottlenecked nodes. Some of them triggered multipath routing for congestion problem remediation. More details of congestion control RPL based solutions is given in [32].

\subsection{Design Complexity}

Authors of the aforementioned RPL OF enhancement solutions approaches tried to overcome the standard RPL drawbacks. However, none of the concerned research works raised memory capacity purpose and the ability of the constrained node to support the final enhancement schemes complexity. In fact, the proposed solutions tend to be complex, due to its storage and processing capacity requirements. Resorting to multipath routing compels nodes to support larger routing tables including alternatives path in particular if 
downward routing is enabled. Moreover, fuzzy logic based metric combinations require intensive memory capacity.

Table 4: Synthesis of enhancement schemes capabilities

\begin{tabular}{|c|c|c|c|c|c|}
\hline Proposal & $\begin{array}{l}\text { Cross } \\
\text { layer } \\
\text { design } \\
\end{array}$ & $\begin{array}{l}\text { Multipath } \\
\text { routing }\end{array}$ & $\begin{array}{l}\text { Mobility } \\
\text { support }\end{array}$ & $\begin{array}{l}\text { Scalability } \\
\text { support }\end{array}$ & $\begin{array}{l}\text { Security } \\
\text { consid- } \\
\text { eration } \\
\end{array}$ \\
\hline $\begin{array}{l}\text { DT-RPL } \\
{[12]}\end{array}$ & $\sqrt{ }$ & $\mathrm{x}$ & $\mathrm{x}$ & - & $\mathrm{x}$ \\
\hline $\begin{array}{l}\text { Di } \\
\text { Marco } \\
\text { et al. } \\
{[13]}\end{array}$ & $\sqrt{ }$ & $\mathrm{x}$ & $\mathrm{x}$ & - & $\mathrm{x}$ \\
\hline $\begin{array}{l}\text { Gonizzi } \\
\text { et al. } \\
{[14]}\end{array}$ & $\sqrt{ }$ & $\mathrm{x}$ & $\mathrm{x}$ & - & $\mathrm{x}$ \\
\hline $\begin{array}{l}\text { RA- } \\
\text { EEDEM } \\
{[15]}\end{array}$ & $\sqrt{ }$ & $\mathrm{x}$ & $\mathrm{x}$ & - & $\mathrm{x}$ \\
\hline $\begin{array}{l}\text { Karkazis } \\
\text { et al. } \\
{[16]}\end{array}$ & $\sqrt{ }$ & $\mathrm{x}$ & $\mathrm{x}$ & $\sqrt{ }$ & $\sqrt{ }$ \\
\hline $\begin{array}{l}\text { Kamgueu } \\
\text { et al. } \\
{[17]}\end{array}$ & $\sqrt{ }$ & $\mathrm{x}$ & $\mathrm{x}$ & - & $\mathrm{x}$ \\
\hline $\begin{array}{l}\text { Iova et } \\
\text { al. [18] }\end{array}$ & $\sqrt{ }$ & $\sqrt{ }$ & $\mathrm{x}$ & - & $\mathrm{x}$ \\
\hline $\begin{array}{l}\text { LB-OF } \\
{[19]}\end{array}$ & $\sqrt{ }$ & $\mathrm{x}$ & $\mathrm{x}$ & $\sqrt{ }$ & $\mathrm{x}$ \\
\hline $\begin{array}{l}\text { BD- } \\
\text { RPL } \\
\text { [20] }\end{array}$ & $\sqrt{ }$ & $\mathrm{x}$ & $\mathrm{x}$ & $\mathrm{x}$ & $\mathrm{x}$ \\
\hline $\begin{array}{l}\text { CA-OF } \\
{[22]}\end{array}$ & $\sqrt{ }$ & $\mathrm{x}$ & $\mathrm{x}$ & $\sqrt{ }^{*}$ & $\mathrm{x}$ \\
\hline $\begin{array}{l}\text { Qu-RPL } \\
\text { [23] }\end{array}$ & $\sqrt{ }$ & $\mathrm{x}$ & $\mathrm{x}$ & - & $\mathrm{x}$ \\
\hline $\begin{array}{l}\text { En-RPL } \\
{[24]}\end{array}$ & $\sqrt{ }$ & $\sqrt{ }$ & $\mathrm{x}$ & $\mathrm{x}$ & $\mathrm{x}$ \\
\hline $\begin{array}{l}\text { IRPL } \\
\text { [25] }\end{array}$ & $\sqrt{ }$ & $\sqrt{ }$ & $\mathrm{x}$ & $\mathrm{x}$ & $\mathrm{x}$ \\
\hline $\begin{array}{l}\text { Kamgueu } \\
\text { et al. } \\
{[27]}\end{array}$ & $\sqrt{ }$ & $\mathrm{x}$ & $\mathrm{x}$ & $\mathrm{x}$ & $\mathrm{x}$ \\
\hline $\begin{array}{l}\text { OF-FL } \\
{[28]}\end{array}$ & $\sqrt{ }$ & $\mathrm{X}$ & $\mathrm{X}$ & $\sqrt{ }$ & $\mathrm{x}$ \\
\hline $\begin{array}{l}\text { OF-EC } \\
{[29]}\end{array}$ & $\sqrt{ }$ & $\mathrm{X}$ & $\mathrm{x}$ & $\sqrt{ }^{*}$ & $\mathrm{x}$ \\
\hline $\begin{array}{l}\text { CMOF } \\
{[30]}\end{array}$ & $\sqrt{ }$ & $\mathrm{x}$ & $\mathrm{x}$ & $\mathrm{x}$ & $\mathrm{x}$ \\
\hline $\begin{array}{l}\text { FMOF } \\
\text { [40] }\end{array}$ & $\sqrt{ }$ & $\mathrm{X}$ & $\sqrt{ }$ & $\mathrm{x}$ & $\mathrm{X}$ \\
\hline $\begin{array}{l}\text { OPP-FL } \\
\text { [31] }\end{array}$ & $\sqrt{ }$ & $\mathrm{x}$ & $\mathrm{x}$ & $\sqrt{ }$ & $\mathrm{x}$ \\
\hline
\end{tabular}

*Slight variation of nodes number

\subsection{Link Reliability and Security Purpose}

Link reliability is a main concern in LLN usually addressed through cross layer design by invoking MAC metrics. However, packet loss is not entirely due to link propriety. It would be interesting to consider security related issues when seeking for PDR optimization. For instance, a non cooperative node may silently drop data instead of forwarding it. If such malicious nodes had a forward position among RPL tree, many of attached nodes can be discarded. In such cases relying only on RPL self-healing mechanisms is insuffisant. We recommend further studies to consider security purposes among major design factors such as application requirement and mobility. In [16] authors started working on combining security requirements (trustworthiness) and link reliability for OF design. More focus on the RPL security enhancement field is provided by [7].

\subsection{Network Scalability}

Scarce are research works that have efficiently addressed scalability concerns while validating their proposed $\mathrm{OF}$ through simulations. In fact, most proposed OF experimentation were made with networks topologies with under fifty nodes. Thus, the proposed schemes are likely to face genuine adaptability issues in bi-directional largescale networks especially with LLNs rising areas of application and IoT. Furthermore, as recommendation for new schemes design, we subjoin thinking about devices heterogeneity and applications interoperability among different hardware configurations.

\section{Opportunities and Future Work}

Most of aforementioned enhancement schemes, were evaluated by referring only to the standard OF defined by the ROLL working group. Thus we face a panoply of objective functions without really having feedback on the effectiveness of one function in relation to another. At this stage, it would be more interesting to work on a new standard that takes into account these different approaches and facilitates their implementation. Thus future proposed $\mathrm{OF}$ will no longer have to refer to a very basic objective function i.e. MRHOF, but rather reinforce already established efforts.Moreover, it would be interesting in the new standard to update the structure of DIO messages so as to intuitively propagate information regarding widely used metrics that have proven their effectiveness such as the number of children attached to a node or the hop count. Thus the effort of research will be focused on calculation methods and optimization algorithms.

The use of AI, particularly Machine Learning (ML) techniques, could be very also interesting insofar as it will make it possible to merge different proposed routing schemes. Researchers can get through ML useful insights to understand which routing metric is more likely to affect network behaves and ensures better performances. Moreover, better adaptability of proposed algorithms to IoT specific applications would be achievable. The ML-based approach will also take advantage of the reduced cost of simulation compared to real test deployment and then allow large-scale networks testing.

In another case, the majority of improvement approaches focus on non-root nodes so it is high time to work on sink nodes by 
Table 5: Synthesis of enhancement schemes achievements

\begin{tabular}{|c|c|c|c|c|}
\hline Proposal & Reliability enhancement & Delay enhancement & Extending lifetime & $\begin{array}{l}\text { Load balancing and conges- } \\
\text { tion avoidance }\end{array}$ \\
\hline DT-RPL [12] & $\sqrt{ }$ & - & $\sqrt{ }$ & - \\
\hline Di Marco et al. 13 & $\sqrt{ }$ & - & $\sqrt{ }$ & $\sqrt{ }$ \\
\hline Gonizzi et al. [14] & - & $\sqrt{ }$ & - & - \\
\hline RA-EEDEM [15] & $\sqrt{ }$ & $\sqrt{ }^{*}$ & - & - \\
\hline Karkazis et al. [16 & $\sqrt{ }$ & - & - & - \\
\hline Kamgueu et al. 17 & - & - & $\sqrt{ }$ & - \\
\hline Iova et al. 18 & $\sqrt{ }^{*}$ & $\mathrm{x}$ & $\sqrt{ }$ & $\sqrt{ }$ \\
\hline LB-OF [19] & $\sqrt{ }^{h}$ & - & $\sqrt{ }^{*}$ & $\sqrt{ }^{*}$ \\
\hline BD-RPL [20] & $\sqrt{ }$ & $\sqrt{ }$ & $\sqrt{ }$ & $\sqrt{ }$ \\
\hline CA-OF [22] & $\sqrt{ }$ & - & - & $\sqrt{ }$ \\
\hline Qu-RPL [23] & $\sqrt{ }$ & - & - & $\sqrt{ }$ \\
\hline En-RPL [24] & $\sqrt{ }$ & $x$ & $\sqrt{ }$ & $\sqrt{ }$ \\
\hline IRPL 25 & $\sqrt{ }$ & $\sqrt{ }^{*}$ & $\sqrt{ }$ & $\sqrt{ }$ \\
\hline Kamgueu et al. [27] & $\sqrt{ }$ & $\sqrt{ }^{*}$ & $\sqrt{ }$ & - \\
\hline OF-FL [28] & $\sqrt{ }$ & $\sqrt{ }$ & $\sqrt{ }$ & - \\
\hline OF-EC [29] & $\sqrt{ }$ & - & $\mathrm{x}$ & - \\
\hline CMOF [30] & $\sqrt{ }$ & $\sqrt{ }$ & - & $\sqrt{ }^{a}$ \\
\hline OPP-FL [31] & $\sqrt{ }^{c}$ & $\sqrt{ }^{c}$ & - & - \\
\hline FMOF 40$]$ & $\sqrt{ }^{b}$ & $\sqrt{ }$ & - & - \\
\hline
\end{tabular}

*No or slight variation

${ }^{h}$ In case of high number of nodes

${ }^{a}$ enhanced through a TX power control mechanism

${ }^{b}$ only in low transmission rate

${ }^{c}$ Significant improve can be observed in case of random node

deployment and high density networks 
optimizing the placement of the collector nodes. Thereby, intermediate nodes can be relieved from complex algorithms involving considerable storage and processing capacity. Indeed the 6LBR is main-powered and benefits from a significant processing and storage capacity which will allow it to deploy quite relevant optimization solutions without worrying about the complexity of the processing involved. In the light of the obtained results, an update will be broadcast through the IPv6 control messages to large networks to invite the other nodes to adapt the new routing parameters promising better performance.

Another big challenge to be faced by RPL is to attest its willing to fit specific IoT application. Although, it has proven its efficiency in smart grid Advanced metering infrastructure (AMI) application, more work is to do in terms of security, reliability, and delay to meet other IoT applications requirements.

\section{Conclusion}

Through this work, we concentrated on RPL enhancement efforts made through the OF as the core of the RPL routing function. We gave an overview of enhancements work made till now to supply LLNS main applications requirements and overcome nodes limitation in terms of energy. First, we exposed a glimpse of the RPL protocol in terms of design, DODAG construction and different traffic patterns. Then, we highlighted researchers endeavor to enhance RPL protocol. Notably, we reviewed a set of literature works tackling major RPL OF improvements. In particular, our survey classified them to single metric based OF enhancement and metric combinations based OF enhancement schemes. For single metric based OF enhancement works, we focus on novel metrics not already tackled in [5]. For metrics combination based OF, which is the widespread technique, we presented weighted linear combination techniques and fuzzy logic based combination techniques. We have also noticed that besides cross-layer design approaches, multipath routing was widely used by a notable number of papers. We also emphasized the need for future research works to focus on the ability of the proposed schemes to apply for networks scalability purpose and real implementation and experiments.

\section{Conflict of Interest}

The authors declare no conflict of interest.

\section{References}

[1] T. Winter et al., "RPL: IPv6 routing protocol for low-power and lossy networks," Internet Eng. Task Force, Fremont, CA, USA, RFC 6550, Mar. 2012.

[2] E.K. Pister , E.P. Thubert, S. Dwars , T. Phinney , "Industrial Routing Requirements in Low Power and Lossy Networks", IETF Request for Comments : 5673, MARCH 2009.

[3] P. Thubert, "Objective function zero for the routing protocol for low-power and lossy networks (RPL)", IETF Request for comments: 6552,March 2012.

[4] O. Gnawali, P. Levis, The minimum rank with hysteresis objective function, IETF request for comments: 6719, September 2012.

[5] J. Vasseur , M. Kim , K. Pister , D. Barthel , 'Routing metrics used for path calculation in low-power and lossy networks", RFC 6551, 1-12, 2012.
[6] E. Ancillotti, C. Vallati, R. Bruno, E.Mingozzi, "A reinforcement learning-based link quality estimation strategy for RPL and its impact on topology management" Computer Communications, 112(3), 1-13, 2017. https://doi.org/10.1016/j.comcom.2017.08.005

[7] P. O. Kamgueu, E. Nataf, T. D. Ndie, "Survey on RPL enhancements:a focus on topology, security and mobility", Computer Communications, 120, 1-17, 2018. https://doi.org/10.1016/j.comcom.2018.02.011

[8] J. V. V. Sobral, J. J. P. C Rodrigues, R. A. L. Rabêlo, J. Al-Muhtadi, V. Korotaev, "Routing Protocols for Low Power and Lossy Networks in Internet of Things Applications" Sensors, 19(9), 21-44, 2019. https://doi.org/10.3390/s19092144

[9] P. Levis , T. Clausen , J. Hui , O. Gnawali , J. Ko , "The Trickle algorithm", IETF RFC 6206, 2011.

[10] P. Levis , N. Patel , D. Culler, S. Shenker ,"Trickle: a self-regulating algorithm for code propagation and maintenance in wireless sensor networks", 1st conference on Symposium on Networked Systems Design and Implementation (NSDI), USA, 2004. https://doi.org/10.5555/1251175.1251177

[11] H. Kharrufa, H. A. A. Al-Kashoash and A. H. Kemp, "RPL-Based Routing Protocols in IoT Applications: A Review," in IEEE Sensors Journal, 19(15), 5952-5967, August, 2019. https://doi.org/10.1109/JSEN.2019.2910881.

[12] H. S. Kim, H. Cho, H. Kim, S. Bahk, 'DT-RPL: Diverse Bidirectional Traffic Delivery through RPL Routing Protocol in Low Power and Lossy Networks" Computer Networks. , 126, 150-161, 2017. https://doi.org/10.1016/j.comnet.2017.07.001

[13] P. Di Marco, C. Fischione, G. Athanasiou and P. Mekikis, "MAC-aware routing metrics for low power and lossy networks," IEEE INFOCOM, Turin, 13-14, 2013. https://doi.org/10.1109/INFCOM.2013.6566722.

[14] P. Gonizzi, R. Monica and G. Ferrari, "Design and evaluation of a delayefficient RPL routing metric" in 2013 9th International Wireless Communications and Mobile Computing Conference (IWCMC), Sardinia, 1573-1577, 2013. https://doi.org/10.1109/IWCMC.2013.6583790

[15] P. Pinto, A. Pinto and M. Ricardo, "RPL modifications to improve the endto-end delay estimation in WSN" in 2014 11th International Symposium on Wireless Communications Systems (ISWCS), Barcelona, 868-872, 2014. https://doi.org/10.1109/ISWCS.2014.6933475.

[16] P. Karkazis, I. Papaefstathiou, L. Sarakis, T. Zahariadis, T. Velivassaki and D. Bargiotas, "Evaluation of RPL with a transmission countefficient and trust-aware routing metric" in 2014 IEEE International Conference on Communications (ICC), Sydney, NSW, 550-556, 2014. https://doi.org/10.1109/ICC.2014.6883376.

[17] P. O. Kamgueu, E. Nataf, T. N. Djotio, O. Festor, "Energy-based metric for the routing protocol in low-power and lossy network", International Conference on Sensor Networks, Barcelona, Spain, 145-148, 2013. https://doi.org/10.5220/0004313401450148.

[18] O. Iova , F. Theoleyre, T. Noel , "Using multiparent routing in RPL to increase the stability and the lifetime of the network", Ad Hoc Netw,29,45-62, 2015. https://doi.org/10.1016/j.adhoc.2015.01.020

[19] M. Qasem, A. Al-Dubai, I. Romdhani, B. Ghaleb and W. Gharibi, "A new efficient objective function for routing in Internet of Things paradigm" IEEE Conference on Standards for Communications and Networking (CSCN), Berlin, 1-6, 2016. https://doi.org/10.1109/CSCN.2016.7785168.

[20] F. Boubekeur, L. Blin, R. Leone, and P. Medagliani, ”Bounding Degrees on RPL" in 2015 ACM 11th Symposium on QoS and Security for Wire less and Mobile Networks (Q2SWinet), New York, USA, 123-130, 2015. https://doi.org/10.1145/2815317.2815339

[21] A. M. A. Awad, R. A. Rahim and A. H. A. Hashim, "Queue Backlog as a Node Metric for RPL Protocol" in 2016 International Conference on Computer and Communication Engineering (ICCCE), Kuala Lumpur, 246-250, 2016. https://doi.org/10.1109/ICCCE.2016.61

[22] H. A. A. Al-Kashoash, Y. Al-Nidawi and A. H. Kemp, "Congestion-aware RPL for 6LOWPAN networks" in 2016 Wireless Telecommunications Symposium (WTS), London, 1-6, 2016. https://doi.org/10.1109/WTS.2016.7482026.

[23] H. Kim, J. Paek and S. Bahk, "QU-RPL: Queue utilization based RPL for load balancing in large scale industrial applications" in 2015 IEEE 12th Annual International Conference on Sensing, Communication, and Networking (SECON), Seattle, WA, 265-273, 2015. https://doi.org/10.1109/SAHCN.2015.7338325.

[24] P. Singh and Y. Chen, 'RPL Enhancement for a Parent Selection Mechanism and an Efficient Objective Function"IEEE Sensors Journal., 19(21), 10054 10066, 2019. https://doi.org/10.1109/JSEN.2019.2927498 
[25] Z. Wang, L. Zhang, Z. Zheng and J. Wang, "An Optimized RPL Protocol for Wireless Sensor Networks"in 2016 International Conference on Parallel and Distributed Systems (ICPADS), Wuhan, 294-299, 2016. https://doi.org/10.1109/ICPADS.2016.0047.

[26] L. A. Zadeh, "Fuzzy logic",Computer,21(4), 83-93,April 1988. https://doi.org/10.1109/2.53.

[27] P. Kamgueu, E. Nataf and T. Ndie Djotio, "On design and deployment of fuzzybased metric for routing in low-power and lossy networks," IEEE 40th Local Computer Networks Conference Workshops (LCN Workshops), Clear water Beach, FL, 789-795, 2015. https://doi.org/10.1109/LCNW.2015.7365929.

[28] O. Gaddour, A. Koubâa, N. Baccour and M. Abid, "OF-FL: QoSaware fuzzy logic objective function for the RPL routing protocol," 12th International Symposium on Modeling and Optimization in Mobile, Ad Hoc, and Wireless Networks (WiOpt), Hammamet, 365-372, 2014. https://doi.org/10.1109/WIOPT.2014.6850321.

[29] H. Lamaazi and N. Benamar, "RPL enhancement using a new objective function based on combined metrics," 13th International Wireless Communications and Mobile Computing Conference (IWCMC), Valencia, 1459-1464, 2017. https://doi.org/10.1109/IWCMC.2017.7986499.

[30] T. G. Harshavardhana, B. S. Vineeth, S. V. R. Anand and M. Hegde, "Power control and cross-layer design of RPL objective function for low power and lossy networks," 10th International Conference on Communication Systems \& Networks (COMSNETS), Bengaluru, 214-219, 2018. https://doi.org/10.1109/COMSNETS.2018.8328200.

[31] I. Kechiche, I. Bousnina and A. Samet, "A novel opportunistic Fuzzy logic based objective function for the Routing Protocol for Low-Power and Lossy Networks," 15th International Wireless Communications \& Mobile Computing Conference (IWCMC), Tangier, Morocco, 698-703, 2019. https://doi.org/10.1109/IWCMC.2019.8766691.

[32] S. Moeller, A. Sridharan, B. Krishnamachari, O. Gnawali, ”Routing without routes: the backpressure collection protocol" in 2010 ACM/IEEE 9th conference on Information Processing in Sensor Networks (IPSN), New York, NY, USA, 279-290, 2010. https://doi.org/10.1145/1791212.1791246
[33] J. Eriksson, F. Österlind, N. Finne, N. Tsiftes, A. Dunkels, T. Voigt, R. Sauter, and P. Marrón,"COOJA/MSPSim: interoperability testing for wireless sensor networks" in 2009 International Conference on Simulation Tools and Techniques (ICSTT),Brussels, BEL, 27, 2009. https://doi.org/2009.10.4108/ICST.SIMUTOOLS2009.5637

[34] P. Pinto, A. Pinto and M. Ricardo, "End-to-end delay estimation using RPL metrics in WSN" in 2013 IFIP Wireless Days (WD), Valencia, 1-6, 2013. https://doi.org/10.1109/WD.2013.6686524

[35] A. Sobeih et al., "J-Sim: a simulation and emulation environment for wireless sensor networks," IEEE Wireless Communications,13(4), 104-119, 2006. https://doi.org/10.1109/MWC.2006.1678171.

[36] E. Ben Hmida, "WSNet Simulator : An event-driven simulator for large scale wireless sensor networks", available online http://wsnet.gforge.inria.fr/download.html”.

[37] L. Chang, T. Lee, S. Chen and C. Liao, "Energy-Efficient Oriented Routing Algorithm in Wireless Sensor Networks," in 2013 IEEE International Conference on Systems, Man, and Cybernetics, Manchester, UK 3813-3818, 2013. https://doi.org/10.1109/SMC.2013.651

[38] E.Fleury, N.Mitton, T.Noel, C.Adjih., "FITIoT-LAB: The Largest IoT Open Experimental Testbed" in ERCIM News, 14, 2015. https://hal.inria.fr/hal01138038

[39] F. Demicheli, G.Ferrari, P. Gonizzi, Design, Implementation and Evaluation of an energy RPL routing metric: Study of an energy efficient routing metric, LAP LAMBERT Academic Publishing, 2014.

[40] I. H. Urama, H. Fotouhi and M. M. Abdellatif, "Optimizing RPL Objective Function for Mobile Low-Power Wireless Networks," IEEE 41st Annual Computer Software and Applications Conference (COMPSAC), Turin, 678-683, 2017. https://doi.org/10.1109/COMPSAC.2017.185.

[41] H. Fotouhi, D. Moreira, and M. Alves, "mRPL: Boosting mobility in the Internet of Things," Ad Hoc Networks, 26, 17-35, 2015. https://doi.org/10.1016/j.adhoc.2014.10.009 